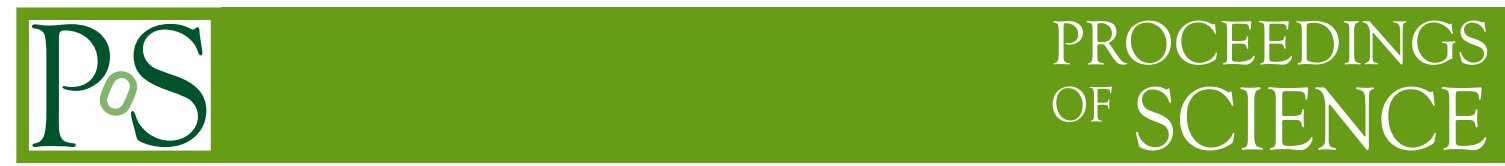

\title{
Search for the Higgs boson at the ATLAS experiment
}

\author{
Elias Coniavitis* \\ on behalf of the ATLAS Collaboration \\ Uppsala University ${ }^{\dagger}$ \\ E-mail: elias.coniavitisecern.ch
}

The search for the Higgs boson is one of the main goals of the ATLAS experiment. A consequence of the Higgs Mechanism for electroweak symmetry breaking, the Higgs boson is the only particle predicted by the Standard Model not yet experimentally discovered. This paper presents an overview of the search channels and expected sensitivity of the ATLAS experiment, both for the Standard Model Higgs boson and for Higgs bosons appearing in supersymmetric extensions.

XVIII International Workshop on Deep-Inelastic Scattering and Related Subjects April 19 -23, 2010

Convitto della Calza, Firenze, Italy

\footnotetext{
*Speaker.

${ }^{\dagger}$ Present affiliation: University of Oxford
} 


\section{Introduction}

The Higgs boson is a consequence of the Higgs Mechanism [1], responsible for breaking the electroweak symmetry and giving mass to the particles of the Standarad Model (SM). It is the only particle predicted by the SM not yet discovered, and to discover or exclude it is one of the principal goals of the ATLAS experiment [2] at CERN's Large Hadron Collider [3].

The Higgs boson has been searched for in both electron-positron and hadron collider experiments. At the Large Electron Positron collider (LEP) at CERN, a Higgs boson of a mass below 114.4 GeV has been excluded at 95\% confidence level [ $[$ ]. At the Tevatron collider at Fermilab, the Higgs boson is currently being searched for in proton-antiproton collisions at $\sqrt{s}=1.96 \mathrm{TeV}$ by both the D0 and CDF experiments. Combining the results from both experiments, using an integrated luminosity of $5.4 \mathrm{fb}^{-1}$ and $4.8 \mathrm{fb}^{-1}$ respectively, it has been possible to exclude a SM Higgs boson for a mass-range of $162-166 \mathrm{GeV}$ at $95 \%$ confidence level [5]].

Models for physics beyond the SM often include a non-minimal Higgs sector, such as e.g. the Minimal Supersymmetric extention of the Standard Model (MSSM) which contains three neutral and two charged Higgs bosons. Such Higgs bosons are also searched for in ATLAS.

\section{The Standard Model Higgs boson}

At the LHC, a Higgs boson can be produced (in order of decreasing cross-section) through gluon fusion $(g g \rightarrow H)$, vector boson fusion $(q q \rightarrow q q H)$ or associated production $(q q \rightarrow W H$ or $Z H, g g, q q \rightarrow t t H)$. Depending on the Higgs boson mass, the decays to $b b, \tau \tau, \gamma \gamma, W W$ and $Z Z$ are important for experimental searches. The production cross-sections and branching ratios as a function of the Higgs mass can be seen in Figure 1 .
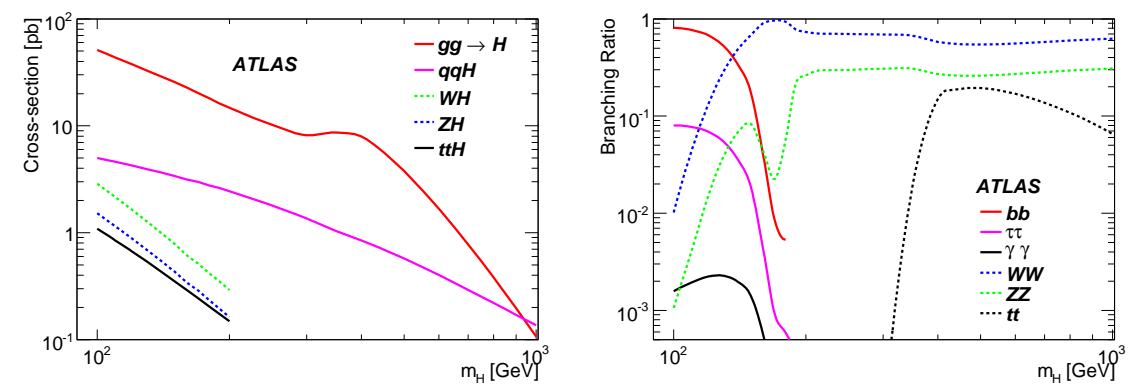

Figure 1: Higgs boson production cross-section at the LHC at $\sqrt{s}=14 \mathrm{TeV}$ (left) and branching ratios (right) as a function of the Higgs mass. [6]

$H \rightarrow b b$ : The Higgs boson decaying to a pair of b-quarks has been studied in two different production modes, $t t H$ and $V H$. The $t t H, H \rightarrow b b$ channel has an experimentally very challenging final state and has been shown to be very sensitive to systematic uncertainties [6]. A very good control of the backgrounds will be necessary for this channel to contribute to the ATLAS discovery sensitivity. The $V H, H \rightarrow b b$ channel was recently investigated at $\sqrt{s}=14 \mathrm{TeV}$ [7] for a highly boosted Higgs boson $\left(p_{T}>200 \mathrm{GeV}\right)$, in which case both b-quarks end up in a single fat jet. 
Analyzing the sub-structure of the fat jet, this approach was shown to substantially improve the sensitivity for $H \rightarrow b b$, while being more robust against systematics than the $t t H$ mode.

$q q H, H \rightarrow \tau \tau: \quad$ Three different channels have been investigated at $\sqrt{s}=14 \mathrm{TeV}$ [6] for the Higgs boson produced in Vector Boson Fusion (VBF) and decaying to a pair of $\tau$ s, depending on whether the $\tau$ decays leptonically or hadronically. This decay mode of the Higgs boson has the second largest branching ratio in the low mass region, and the collinear approximation allows the reconstruction of the Higgs mass, with a mass resolution of $\sim 10 \mathrm{GeV}$. The characteristic topology of VBF is exploited to suppress the most important backgrounds, by demanding two forward jets with a large rapidity gap and applying a central jet veto while at the same time demanding the $\tau$ decay products to be in the central part of the detector. The shape of the most important background, $Z \rightarrow \tau \tau$, can be obtained from data, by replacing real $\mu$ s with simulated $\tau$ s in $Z \rightarrow \mu \mu$ samples.

$H \rightarrow \gamma \gamma$ : Despite its small branching ratio, the decay of the Higgs boson to two $\gamma_{\mathrm{s}}$ is an important channel, as it allows for a clear mass peak to be reconstructed. ATLAS has performed both an inclusive analysis, as well as studying diphotons in association with 1 or 2 jets, at $\sqrt{s}=14 \mathrm{TeV}$ [6]. It was shown that a good mass resolution $\left(\sigma_{m} / m \sim 1.2 \%\right)$ can be obtained. Powerful $\gamma$ identification is required to reduce the backgrounds from jets faking $\gamma_{\mathrm{s}}$ and this is achieved by making use of shower shape and track isolation requirements, while $\gamma$ conversions can be efficiently reconstructed by combining information from the electromagnetic calorimeter and the tracker.

$H \rightarrow W W \rightarrow l v l v: \quad$ ATLAS has investigated the $H \rightarrow W W$ channel in the $H+0$ jets and $H+2$ jets modes for the $e v \mu v$ channel at $\sqrt{s}=14 \mathrm{TeV}$ [ [6], as well as for all three lepton channels $(e e, \mu \mu, e \mu+2 v)$ in 3 jet bins $(H+0,1,2$ jets $)$ at $\sqrt{s}=10 \mathrm{TeV}[8]$. Due to the spin correlations of the $W$-bosons from the $H$, one can discriminate against the background by demanding the presence of two leptons with a small transverse opening angle. In the case of the 2-jet analysis, cuts are made reflecting the characteristic VBF topology (two forward jets with rapidity gap). The two neutrinos in the final state prohibit the reconstruction of a mass peak, so the transverse mass is reconstructed instead. Control regions are used to study the dominant backgrounds with data and extrapolate them into the signal region. Figure 2 (left) shows the expected exclusion potential at $\sqrt{s}=10$ $\mathrm{TeV}$, assuming $200 \mathrm{pb}^{-1}$. This channel is expected to have the earliest sensitivity, for Higgs boson masses around $160 \mathrm{GeV}$.

$H \rightarrow Z^{(*)} Z \rightarrow 4 l$ : The $H \rightarrow Z^{(*)} Z \rightarrow 4 l$ channel is a very promising one, as it allows for a clear peak to be reconstructed on top of a smooth background, while a wide range of masses can be covered. ATLAS has studied the $4 e, 2 e 2 \mu$ and $4 \mu$ modes for $\sqrt{s}=14 \mathrm{TeV}$ [6]. Two pairs of same flavour, opposite sign leptons are demanded from which the $Z, Z^{(*)}$ and $H$ masses are reconstructed. The background is further suppressed by requirements on the lepton isolation and impact parameter. The remaining backgrounds are estimated by making a fit on the sidebands. A mass resolution of $\sim 2-3 \mathrm{GeV}$ can be obtained, while the discovery potential reaches up to very high Higgs masses.

Combination: The combined expected Higgs boson sensitivity reach for ATLAS at $\sqrt{s}=14 \mathrm{TeV}$ was calculated [6] using the profile likelihood ratio method and is shown in Figure 2. As can be seen, already with $2 \mathrm{fb}^{-1}$ at $14 \mathrm{TeV}$, ATLAS has a $5 \sigma$ (or more) discovery sensitivity for the mass range of $143-179 \mathrm{GeV}$ while the exclusion sensitivity at 95\% C.L. reaches as low as $115 \mathrm{GeV}$. 

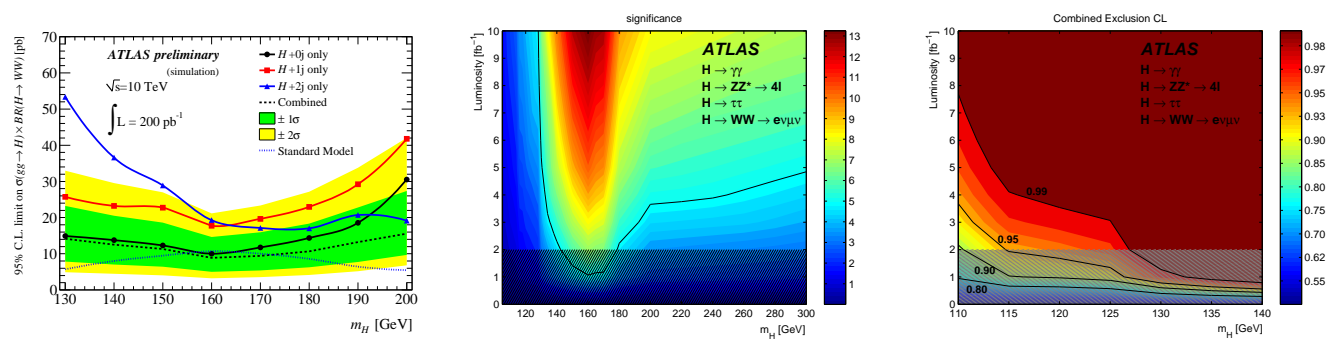

Figure 2: Left: ATLAS expected $95 \%$ confidence level limit on the signal normalization as a function of Higgs boson mass in the $H \rightarrow W W \rightarrow l v l v$ channel, assuming $200 \mathrm{pb}^{-1}$ at $\sqrt{s}=10 \mathrm{TeV}$. Combined results: ATLAS combined discovery (centre) and exclusion (left) potential for Standard Model Higgs boson searches at $14 \mathrm{TeV}$ centre-of-mass energy.

\section{MSSM Higgs bosons}

In the MSSM, the Higgs sector consists of two Higgs doublets (as opposed to one in the SM) which leads to five physical Higgs bosons $\left(h, H, A, H^{ \pm}\right)$. At tree level, this Higgs sector can be described using only two parameters, conventionally $m_{A}$ and $\tan \beta$. The discovery potential for both neutral and charged Higgs bosons was evaluated in the $m_{h}^{\max }$ scenario [9] of the MSSM.

For the neutral Higgs bosons of the MSSM the dominant production modes are gluon fusion and associated production with b-quarks. Decays to third generation fermions are enhanced with respect to the SM, while decays to vector bosons are suppressed $(h, H)$ or completely absent $(A)$.

The $h / H / A \rightarrow \mu \mu$ decay has been studied by ATLAS for $\sqrt{s}=14 \mathrm{TeV}$ in both the $0 \mathrm{~b}$-tag (i.e. gluon-fusion production) and the $\geq 1 \mathrm{~b}$-tag (associated production) channels [6]. The dominant backgrounds are estimated using sidebands and control samples (e.g. using the ee channel to get a signal-free control region). A mass resolution around 6-7 GeV can be achieved. Figure 3 (left) shows the expected discovery potential of this channel.

ATLAS has also studied the $h / H / A \rightarrow \tau \tau \rightarrow l l+4 v$ channel in associated production, requiring at least one b-tagged jet [6]. The main backgrounds are $Z \rightarrow \tau \tau$ for low and $t t$ for high Higgs boson masses. The $Z \rightarrow \tau \tau$ background shape and normalization is estimated from $Z \rightarrow \mu \mu / e e$ sideband events. The mass can be obtained using the collinear approximation, yielding a resolution of around $25 \mathrm{GeV}$ in the low-mass region and around $80 \mathrm{GeV}$ in the high-mass region. The expected discovery potential of this channel is shown in Figure 3 (centre).

The charged Higgs bosons are primarily produced in top quark decays if $m_{H^{ \pm}}<m_{t}$, else in $g g / g b$-fusion. For low masses the decay $H^{ \pm} \rightarrow \tau \nu$ is the most important, while $H^{ \pm} \rightarrow t b$ starts to dominate when it becomes kinematically accesible.

Five different $H^{ \pm}$analyses have been performed by ATLAS [6] at $\sqrt{s}=14 \mathrm{TeV}$ : three studying the $t t \rightarrow b W b H^{ \pm}, H^{ \pm} \rightarrow \tau \nu$ process, with $\tau \rightarrow l v v$ or hadrons and $W \rightarrow l v$ or $q q$; and two studying $g g / g b \rightarrow t[b] H^{ \pm}$with $H^{ \pm} \rightarrow t b$ or $H^{ \pm} \rightarrow \tau_{\text {had }} v$. The latter is the most important channel in the high-mass region, while for a low $H^{ \pm}$mass the $t t \rightarrow b q q b H^{ \pm}, H^{ \pm} \rightarrow \tau_{\text {had }} v$ has the highest expected sensitivity. Due to the complicated final states, good $\tau$ - and b-tagging as well as missing transverse energy and lepton reconstruction are necessary. The most important background, $t t$, can be studied from data using a $\tau$-embedding technique similar to that used in the SM VBF $H \rightarrow \tau \tau$ analysis. 
Figure 3 (right) shows the expected discovery potential for charged Higgs bosons after combining all channels.
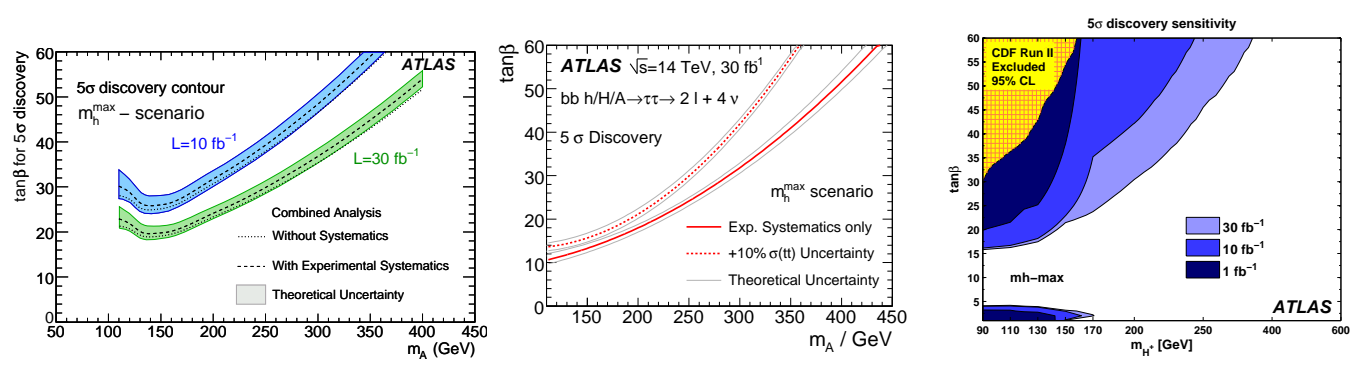

Figure 3: ATLAS discovery potential at $\sqrt{s}=14 \mathrm{TeV}$ for the $h / H / A \rightarrow \mu \mu$ (left) and $h / H / A \rightarrow \tau \tau \rightarrow$ $l l+4 v$ (centre) channels, and the combined $H^{ \pm}$discovery contour (right).

\section{Conclusions}

The discovery of the Higgs boson is one of the main goals of the ATLAS experiment. The expected sensitivity for a large number of channels has been investigated both for the SM Higgs boson and for MSSM Higgs bosons. While it has been shown that ATLAS has sensitivity for a Higgs boson also at lower centre-of-mass energies, most of these studies have been performed for $\sqrt{s}=14 \mathrm{TeV}$ where the full Higgs boson search potential will be reached. ATLAS expects to be sensitive to a SM Higgs boson over the full mass-range, while for MSSM Higgs bosons a substantial part of the $m_{A}-\tan \beta$ plane is expected to be covered.

\section{References}

[1] P. Higgs, Broken Symmetries and the Mass of the Gauge Bosons, Phys. Rev. Lett. 13 (1964) 508-509.

[2] ATLAS Collaboration, G. Aad et. al., The ATLAS Experiment at the CERN Large Hadron Collider, JINST 3 (2008) S08003.

[3] LHC Yellow Book. CERN, 1995. CERN-AC-95-05.

[4] LEP Working Group for Higgs boson searches, R. Barate et. al., Search for the standard model Higgs boson at LEP, Phys. Lett. $\mathbf{B 5 6 5}$ (2003) 61-75, hep-ex/0306033].

[5] CDF and D0 Collaborations, T. Aaltonen et. al., Combination of Tevatron searches for the standard model Higgs boson in the W+W-decay mode, arXiv:1001.4162].

[6] ATLAS Collaboration, G. Aad et. al., Expected Performance of the ATLAS Experiment - Detector, Trigger and Physics, arXiv:0901.0512]. CERN-OPEN-2008-020.

[7] ATLAS Collaboration, ATLAS Sensitivity to the Standard Model Higgs in the HW and HZ Channels at High Transverse Momenta, ATL-PHYS-PUB-2009-088.

[8] ATLAS Collaboration, Prospects for Higgs Boson Searches using the $H \rightarrow W W^{(*)} \rightarrow \ell v \ell v$ Decay Mode with the ATLAS Detector for $10 \mathrm{TeV}$, ATL-PHYS-PUB-2010-005.

[9] ALEPH, DELPHI, L3 and OPAL Collaborations, S. Schael et. al., Search for neutral MSSM Higgs bosons at LEP, Eur. Phys. J. C47 (2006) 547-587, [hep-ex/0602042]. 